\title{
Neural Network-Based System Identification for Quadcopter Dynamic Modeling: A Review
}

\author{
Mohammad Fahmi Pairan ${ }^{1}$, Syariful Syafiq Shamsudin ${ }^{1^{*}}$, Mohd Fadhli \\ Zulkafli $^{1}$
}

${ }^{1}$ Department of Aeronautical Engineering, Faculty of Mechanical and Manufacturing Engineering, Universiti Tun Hussein Onn Malaysia (UTHM), Parit Raja, 86400 Batu Pahat, Johor, MALAYSIA

*Corresponding Author

DOI: https://doi.org/10.30880/jamea.2020.01.02.003

Received 18 August 2020; Accepted 26 October 2020; Available online 31 October 2020

\begin{abstract}
A quadcopter is a rotorcraft with a simple mechanical construction. It has the same hovering capability similar to the traditional helicopter, but it is easier to maintain. The quadcopter is hard to control due to its unstable system with highly coupled and non-linear dynamics. In order to design a robust control algorithm, it is crucial to obtain a precise quadrotor flight dynamics through system identification approach. System identification is a method of finding the mathematical model of the dynamics system using the input-output data measurement. Neural network (NN) based system identification is excellent alternative modeling because it reduces development costs and time by avoiding governing equations and large aerodynamic database. NN based system identification has successfully identified the quadcopter dynamics with good accuracy. This paper gives an overview of the characteristic of the quadcopter and presents a comprehensive survey of the modeling techniques used to determine the flight dynamics of a quadrotor with a particular focus on $\mathrm{NN}$ based system identification method. The presented research works have been classified into different categories such as the first principle modeling, system identification and implementation of NN based system identification in quadcopter platform. Finally, the paper highlights challenges that need to be addressed in developing efficient $\mathrm{NN}$ based system identification model for unmanned quadcopter system.
\end{abstract}

Keywords: Quadcopter, System Identification, Neural Network, Multilayer Perceptron, Radial Basis Function

\section{Introduction}

A quadcopter is a type of rotorcraft based Unmanned Aerial Vehicle (RUAV) that consists of four main fixed-pitch rotors. The quadcopter performs aerial maneuvers by changing the speed of each rotor to generate the desired total thrust and torque for turning and flying. Quadcopter does not require any complex control mechanism for its propellers and, thus is much easier to maintain compared to the conventional helicopter UAV. Multirotor aerial vehicles such as quadcopter have been widely used for numerous civil and military applications. The unique flying capabilities of the quadcopter to take off and land vertically (VSTOL), hovering and cruising at a slower speed makes it suitable for monitoring and surveillance over targets compare to the fixed wing UAV. Multi-rotor UAVs are rapidly conquering the market where various multi-rotor designs are produced for the military as well as the civil applications [1]. In the military application, RUAV is mainly used for real-time reconnaissance, surveillance, and search and rescue missions. On the other hand, the quadcopter is often used in aerial photography, delivery services [2], traffic monitoring [3], and structural inspection [4] in the civil application.

Researchers have developed many different fields of studies using a quadcopter, such as flight dynamic modeling [5], flight control theory [2][6][7], navigation [8]-[11], and robotic [12]. Most of the applications mentioned require the quadrotor to have a highly robust control system to hover in steady and close proximity relative to the targets. Hence, 
comprehensive modeling work needs to be conducted to obtain an accurate flight dynamics model if one intends to design a robust flight control system for a quadcopter.

Aircraft flight dynamics modeling is a numerical representation that can either be linear or nonlinear in the steadystate performance and dynamic responses, such as acceleration, angular rate, and position for actuator's input as precisely with flight motion in the real situation [13]. Any unmodeled dynamics from simplifying assumptions that are not assimilated in the mathematical model will reduce the performance of dynamic models [14]. Generally, there are two basic methods to determine the mathematical model: (1) the first principle of modeling based on Newton-Euler formulation law and Euler's angles and (2) system identification. The first principle modeling is based on the physical understanding of forces and moments balance of the vehicle by direct use of the laws of mechanics and aerodynamics to derive the nonlinear model of a quadcopter. A quadcopter's dynamics model also requires some assumptions to simplify the difficulty of the model. High frequency and unmodeled dynamics are ignored to simplify dynamic model analysis.

As a consequence, flight controller architecture based on simplistic and unmodeled dynamics may not perform appropriately in a real application, resulting in a crash or unpredictable control actions during flight [15]-[17]. The dynamics model from the first principle approach derives mainly from a quadcopter's experiments and physical calculations. Changes in quadcopter parameters will influence the current model of dynamics, so further tests and time are needed to establish a new model of dynamics. In order to simplify the labour-intensive and time-consuming first principal modelling equations, system identification method is suggested by several researchers [2], [18]-[21].

System identification method was introduced to determine the unmodelled parameters in the first principle of modeling from the experimental data collected during the flight test. The neural network (NN) method can be used as an alternative technique in quadcopter dynamic modeling. NN does not depend on a mathematical model that is unique [22]. It has a very flexible model structure which can increase the precision of prediction. However, due to multiple models available, the researcher would have trouble deciding the best model structure and initial NN parameter for the quadcopter. Therefore, any a priori information must be used in order to restrict the number of potential models. The modelling outcome from the NN method can be unreliable, takes longer training time, slow convergence rate, and is vulnerable to the over-fitting problem due to incorrect selection of model structure, an excessive number of neurons, and inadequate machine training data [23], [24].

The work presented in this paper attempts to review the development of a system identification method based on the NN model for quadcopter dynamic modeling application. This paper is organized as follows. In Section 2, the basic structure and characteristic of the quadcopter are presented. In Section 3, first-principle modeling of a quadcopter is discussed briefly. System identification of quadcopter, such as time-domain system identification, frequency-domain domain system identification, and NN system identification are presented in Section 4. In Section 5, more details on the application of system identification based on NN in quadcopter are discussed. Finally, the conclusion of this paper is presented in Section 6.

\section{Quadcopter Characteristic}

The structure of a quadrotor is straightforward and usually has two basic types of configurations, i.e. the 'cross' configurations and the 'plus' configurations. A 'cross' configuration quadrotor is more stable and provides higher momentum than the 'plus' configurations, which increases maneuverability performance [25]. Basic quadcopter controls for 'cross' configuration in the body reference frame respect to inertia frame are shown in Fig. 1. Two diagonal rotors (M1 and M3) are rotating counter-clockwise whereas the other rotors (M2 and M4) rotate in the clockwise direction. The change in angular speed between the four rotors causes variances in thrust and consequently, creates tilting movements around the quadcopter axes. $\mathrm{XB}, \mathrm{YB}$, and $\mathrm{ZB}$ are the main axis in the body frame of the quadcopter, while $\mathrm{X}, \mathrm{Y}$, and $\mathrm{Z}$ are the axis on earth frame.

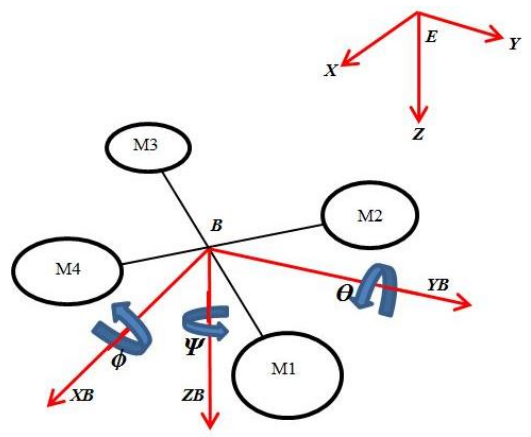

Fig. 1 - Quadcopter free body diagram

The position of the quadcopter can be addressed in the body frame coordinate (B) with reference to earth frame (E). Quadcopter needs to decrease the rotor speed at M1 and M4, and increase the rotor speed at M2 and M3 to move along 
positive XB axis. Thus, the quadcopter will pitch forward with pitch angle $(\theta)$. Similarly, when the quadrotor flies in positive YB axis with roll angle $(\phi)$, the quadcopter needs to decrease rotor speed at M1 and M2 and increase the rotor speed at M3 and M4. In order to make the quadcopter turns in ZB axis with yaw angle $(\psi)$, the quadcopter must increase the speed of M1 and M3 rotors and decrease rotor speed at M2 and M4.

A quadrotor is a highly nonlinear, multivariable, strongly coupled, under-actuated, and an unstable system. It is under-actuated systems due to there are only four parallel force inputs to control its six output coordinates (position and orientation in space). The quadcopter also has a highly coupled system because any change in the speed of one propeller causes another motion of at least three degrees of freedom (DOF ) [26]. Due to the coupled nature of the quadrotor, reducing the speed of one rotor will cause the quadrotor to tilt toward rotor direction, but there are also changes in the total yaw moment and thrust. Hence, all rotor speeds should be controlled for creating any maneuver of quadcopter [4].

\section{First Principle Modeling}

The first principle method of quadrotor modeling is based on a direct physical understanding of forces and moments' balance of the vehicle by using Newton's law and Euler's angles to describe the system behaviour. Generally, the first principle modeling is developed based on established aircraft theories with a certain number of assumptions and simplifications. Many unknown parameters in the mathematical model need to be measured or approximated to make the model work more sophisticatedly. Several assumptions made on a quadcopter to simplify developing mathematical model are as follows: (1) The quadrotor frame is supposedly symmetrical and rigid [2], [27]; (2) The propellers are assumed to be rigid [18]; (3) The center of gravity and center body frame origin is assumed to coincide [28]; and (4) The aerodynamics effects such as flapping and feathering on rotors are ignored [29].

Figure 2 shows the basic flight dynamics model for a quadcopter that represents four main components, which are kinematics, 6 DOF rigid body dynamics, aerodynamic forces and moments and on-board stabilizer dynamics. The overall dynamics model equations for quadcopter are given by

$$
\begin{gathered}
\dot{\mathbf{P}}_{\mathrm{n}}=\mathbf{R}_{\mathrm{n} / \mathrm{b}} \mathbf{V}_{\mathrm{b}} \\
\dot{\boldsymbol{\Phi}}=\boldsymbol{R}_{n / b} \omega_{b} \\
\dot{\mathbf{V}}_{b}=-\boldsymbol{\omega}_{\mathrm{b}} \times \mathbf{V}_{\mathrm{b}}+\frac{\mathbf{F}_{\mathrm{b}}}{m}+\frac{\mathbf{F}_{g}}{\mathrm{~m}} \\
\dot{\boldsymbol{\omega}}_{\mathrm{b}}=\mathbf{J}^{-1}\left[\mathbf{M}_{\mathrm{b}}-\boldsymbol{\omega}_{\mathrm{b}} \times\left(\mathbf{J} \omega_{\mathrm{b}}\right)\right] \\
\mathbf{F}_{\mathrm{b}}=\sum_{\mathrm{i}=1}^{\mathrm{j}} \mathrm{F}_{i} \\
\mathbf{M}_{\mathrm{b}}=\sum_{i=1}^{\mathrm{j}} M_{i} \\
\boldsymbol{\delta}=\left[\boldsymbol{\delta}_{\text {thr }, \boldsymbol{d}}, \boldsymbol{\delta}_{\text {yaw }, \boldsymbol{d}} \boldsymbol{\delta}_{\text {pitch }, \boldsymbol{d}} \boldsymbol{\delta}_{\text {roll, } \boldsymbol{d}}\right]
\end{gathered}
$$

where $\mathbf{P}_{n}=\left[\begin{array}{lll}p_{x} & p_{y} & p_{z}\end{array}\right]^{\prime}$ is the quadcopter position in the inertial reference frame, $\boldsymbol{\Phi}=\left[\begin{array}{lll}\boldsymbol{\phi} & \theta & \psi\end{array}\right]^{\prime}$ is the Euler angles in the body reference frame, $\mathrm{V}_{\mathrm{b}}=\left[\begin{array}{lll}u & v & w\end{array}\right]^{\prime}$ is the linear velocities in the body reference frame, and $\boldsymbol{\omega}_{\mathrm{b}}=\left[\begin{array}{lll}p & q\end{array}\right]^{\prime}$ is the angular rates of the quadcopter in the body reference frame. $\mathbf{R}_{\mathrm{n} / \mathrm{b}}$ is the rotational matrix of body reference to an inertial reference frame, $\mathrm{m}$ is mass of quadcopter, $i$ is the number of the propeller, $\mathrm{J}$ is the moment of inertia matrix for the quadrotor platform, and $\mathbf{F}_{\mathrm{b}}, \mathbf{F}_{g}$ and $\mathbf{M}_{\mathrm{b}}$ are the total applied force to the quadrotor, the gravity force and the total applied moments respectively.

The first principles model of the quadcopter (Equation (1)-(7)) is also known as the lumped parameter approach. By examining all equations involving the first principle modeling, we can conclude that Equation 1 to Equation 7 is a nonlinear model with strongly coupled variables. It can be shown that multiplication between system states and coupling among the variables will create problems in controller design [26], [30]. The first principle modeling for high order nonlinear model will have some unknown ambiguity parameters, although there has enough knowledge about the model itself [14]. The first principal modeling also requires in-depth mathematical and physical calculations about the system behaviour, and it can time-consuming to develop [2], [18], [19]. Hence, system identification was proposed by many researchers to simplify the first principal modeling method. 


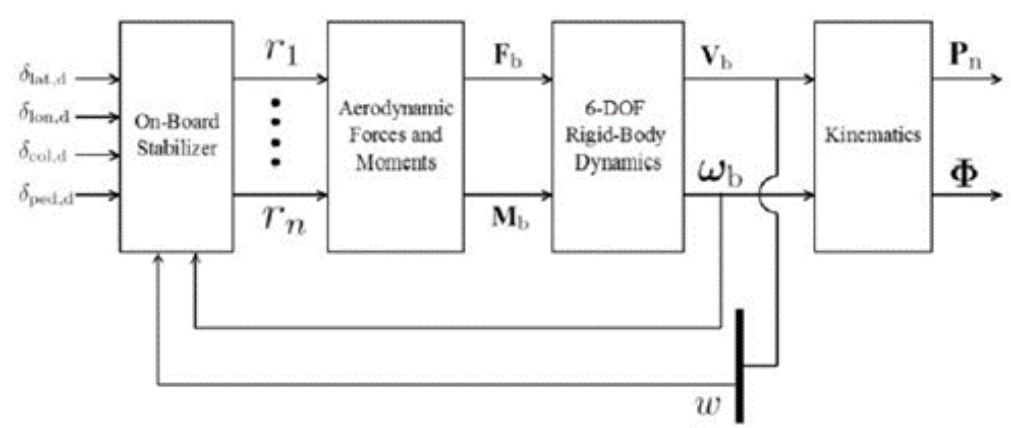

Fig. 2 - Basic dynamics architecture of quadcopter

\section{Time and frequency domain system identification}

System identification is an alternative method to derive dynamic models, which offers the advantages in accuracy and complexity. This technique involves the procedure of deriving a mathematical model based on experimental data from the quadcopter's control inputs and measured outputs. As a result, the flight dynamics model based on the first principal modeling in Fig. 1 can be encapsulated and simplified into the black box dynamic model as in Fig. 3. Therefore, the quadcopter parameters such as the moment of inertia, force, mass, and engine constant are not required, and the complexity of Equations 1 to 6 can be avoided [31], [32].

System identification based on time-domain analysis for a quadcopter was presented by Gremillion and Humbert [33], Miller [34], as well as Yoo and Hong [35]. It involves the use of the transfer function or state-space model of the quadcopter as the model structure, and the model parameters are determined using the linear least-squares method or maximum likelihood method. These methods minimize the cost function and enable the attainment of a dynamic model. It should be noted that the time domain method is preferable for the single input and single-output (SISO) system [30]. However, the quadcopter dynamics system is typically MIMO (multiple-input and multiple outputs). Hence, crosscoupling features in quadcopter must be diminished before the implementation of time-domain system identification [30]. The time-domain system identification is not the best approach for unstable quadrotor because the equations of motion to be numerically integrated with time for each iterative update in the parameters. It also will need a large amount of historical data in the iteration and thus, generate a heavy computational burden to the microcontroller of quadcopter [36].

There is a large volume of published studies [13], [16], [37] on system identification based on the frequency domain. Most of the aforementioned researchers used Comprehensive Identification from Frequency Responses (CIFER) software as an identification tool to implement frequency domain identification. Cai et al. [25] agreed that frequency domain identification is time-consuming and laborious, especially the data collection and pre-processing process. The frequencydomain method requires a longer development time to be implemented since many experiments and simulations are needed to determine the dynamic parameters.

Frequency domain result could be inaccurate and inconsistent because of the insufficient data in very low bandwidth frequency and the removal effect of some dynamic components. Even with the satisfactory identification results from the frequency domain, G. Cai et al. [16] suggested that more focus is needed in data collection and model architecture reconstruction in order to improve the model accuracy further. A complete comparison between frequency and timedomain system identification can be referred to in [20].

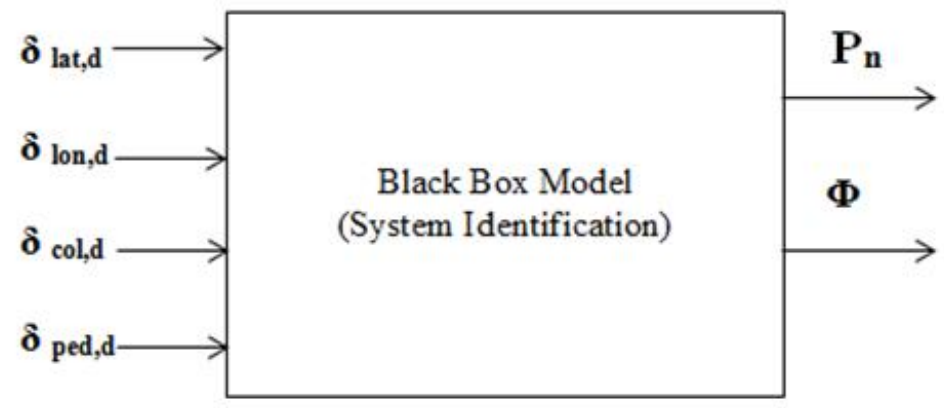

Fig. 3 - System identification based on the black-box model 


\section{Neural network system identification}

System identification based on the NN method can be used as an alternative technique in quadcopter dynamic modeling. Artificial NN is a computational model identical to the structure of biological NN inside the human brain as shown in Fig. 4. Biological neuron gain signals from another neuron through synapses located on the dendrites of the neuron. When the signals received are adequate, the neuron is activated and begins to process signals within its cell body. Then, the processed signals are transferred through the axon to another synapse or another cell body [38]. NN model replicates the similar connection mechanism of biological NN. The modeling capability is achieved through the process of learning as in Fig. 5.

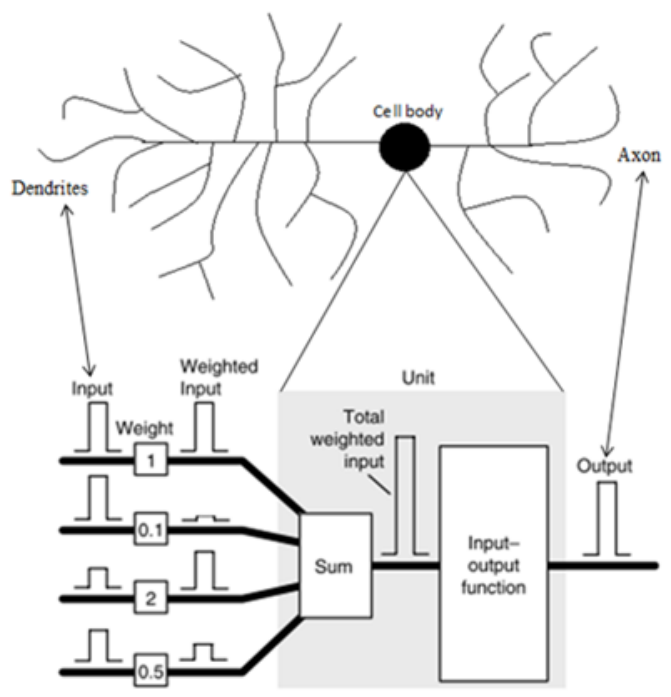

Fig. 4 - Comparison between biological and artificial neural network

NN structure consists of inputs (similar to synapses), which are multiplied by weights (strength of the signals), and a function to computes the output (threshold). As illustrated in Figure 5, $y_{i}$ is the output of neuron $i, x_{j}$ is the $j^{\text {th }}$ input, $w_{i j}$ is the weight given to input $j$ when it is going to neuron $i$ and $w_{i o}$ is the bias of the neuron. The activation function of the neuron $f($.$) can have several forms such as the sigmoid, linear, step or a radial basis function [39]. NN in the real$ application can be implemented to model complex relationships between inputs and outputs. Prediction, pattern recognition, classification, forecasting, and function approximation are the various implementation of NN in real-life applications.

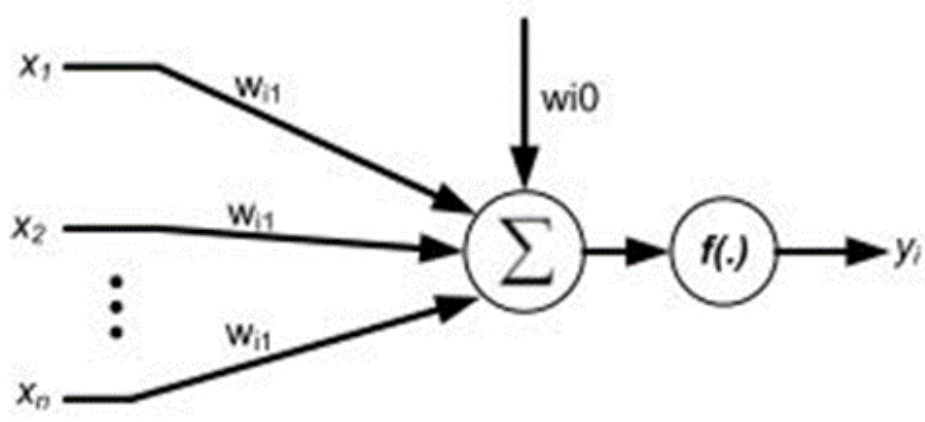

Fig. 5 - Basic Artificial Neural Network model

There are two types of NN structure widely used in system identification; they are feedforward neural (FNN) and recurrent neural network (RNN). In FNN, input information transfers in one direction (forward) only and there are no cycles or loops involved in the network. Contrary to feedforward networks, RNN is NN type models with bidirectional input flow (forward and fed back). The ability of RNN in prediction is limited because of the fixed number of neurons in the state-space formulation. Meanwhile, FNN is capable of estimating any nonlinear function with any level of accuracy [40]. FNN has been successfully implemented to predict the models and parameters of aircraft [14], [41], [42]. 
Fig. 6 shows the general process flow of system identification based on the neural network model. The neural network model is placed in parallel with the actual quadcopter plant. NN model will be trained with inputs and outputs of the quadcopter to predict the output of the plant. Inputs and outputs will represent in time regression vector (past input and output) as training data pair for the neural network model. The output error data between the predicted NN model and actual quadcopter plant will be used to adjust the NN parameters to minimize output error for the next iteration.

NN model approach capable to adapt with nonlinear mapping, robustness, noise resistance, and global approximator that make it a suitable candidate for dynamics modeling [43]-[45]. NN model is a highly flexible model structure with a various number of efficient $\mathrm{NN}$ training and structure optimization algorithms available and can be characterized by several parameters only [46]. System identification based NN model avoids the needs to build extensive aerodynamics database as required in the first principle modeling and time domain-based system identification, thus potentially reduces development costs and time during identification and the modeling process. However, NN modeling may require longer training time, prone to slow convergence rate, and is susceptible to the over-fitting problem if the training was done using a traditional method such as backpropagation method [14].

The training in NN modelling used to minimize the error cost function between the predicted output and the measurement data. The NN modelling can either be trained by using batch or recursive training method. For the batch training method, the NN model learns the relation between the measured inputs and outputs data by selecting the best NN weight over repetitive iteration. The network parameters such are weight and bias of NN offline training updated after the presentation of all training data. Meanwhile, for the recursive training method, the network parameters will continuously be updated based on a single data set to inject into the NN model at the current sample time.

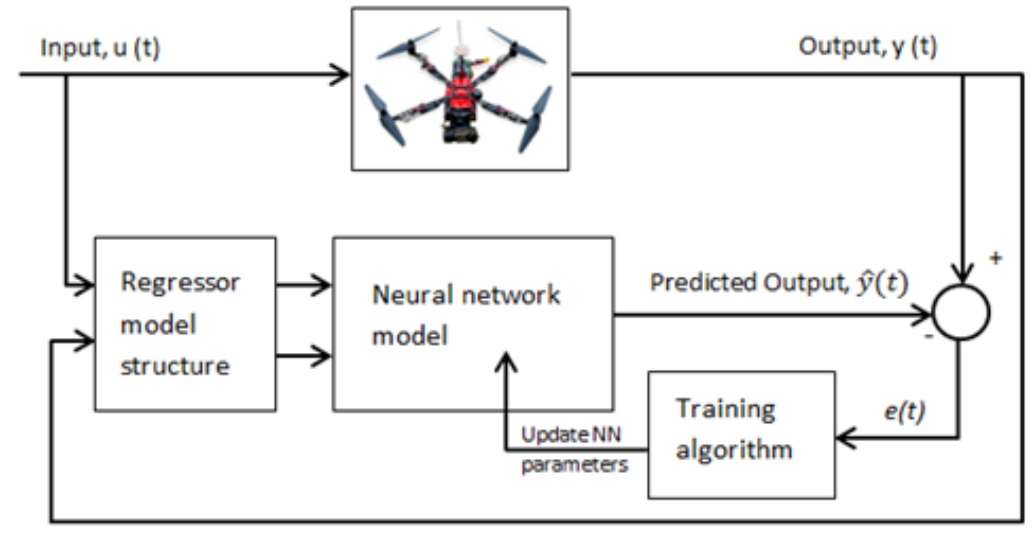

Fig. 6 - System Identification based on neural network

\section{Application of System Identification based on Neural Network for quadcopter}

Although many research works have been carried out on NN system identification, there is a lack of study in the application of $\mathrm{NN}$ based system identification for the multirotor platform. However, there are several examples of the successful study of NN based system identification application for multirotor as shown in Fig. 7 [47]-[50]. This factor further motivated us to find a comprehensive modeling method for a quadcopter, especially in NN based system identification. Two neural network system identification structures for quadcopter are considered in this paper; i.e. the multilayer perceptron (MLP) and radial basis function (RBF). 


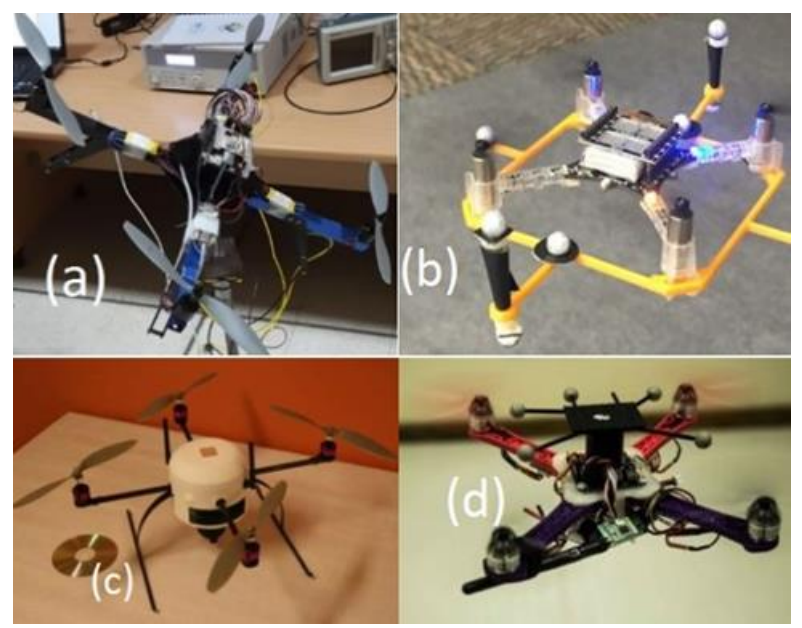

Fig. 7 - Quadcopter platform (a) Arducopter [51]; (b) Crazyflie2.0 [52]; (c) LinkQuad [50]; (d) 3D printer quadrotor [53]

\subsection{Multilayer perceptron structure (MLP)}

MLP is the most common NN architecture used for nonlinear system identification. Fig. 8 depicts the basic architecture of MLP, which consists mainly of one input layer, one or more hidden layer, and one output layer. The output calculation from the MLP structure represents a single hidden layer MLP network as shown:

$$
\hat{y}(t \mid \theta)=g_{h}\left(\sum_{h=1}^{H} w 2_{i h} f_{h}\left(\sum_{j=1}^{m} w 1_{h j} \varphi_{j}+b_{h}\right)+B_{i}\right)
$$

with $\mathrm{h}=1,2,3 . . H$ and $\mathrm{i}=1,2,3, . . n$ where $w 1_{h j}$ are the weights between the input layer and the hidden layer and $w 2_{i n}$ is the weights between the hidden layer and the output layer. The functions $g_{h}$ and $f_{h}$ are nonlinear activation function for neurons in each hidden layers and output layer, respectively. Index $\mathrm{H}$ denotes the number of neurons in the hidden layer while $b_{h}$ and $B_{i}$ are the bias elements for the input layer and the output layer. The number of inputs and outputs of the neural network is presented by $\mathrm{m}$ and $\mathrm{n}$, respectively.

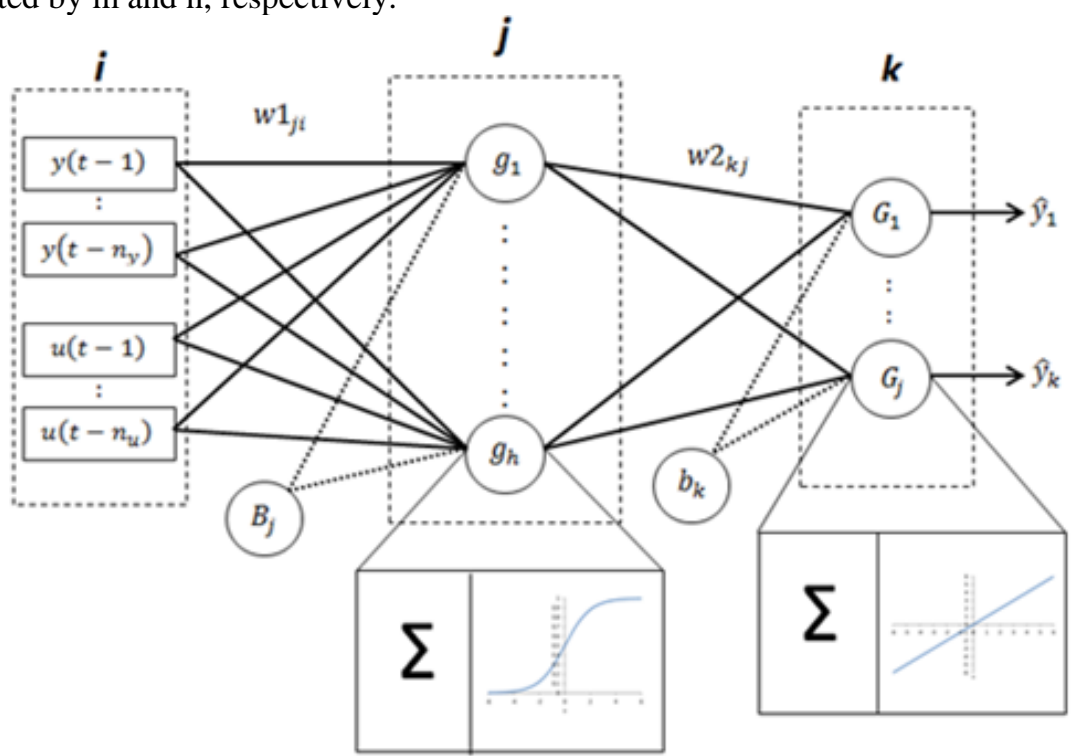

Fig. 8- Basic MLP NN structure

Recent research carried out by Dief and Yoshida [54] involves the use of MLP NN trained with the backpropagation algorithm to identify the system parameter of the quadrotor. The proposed MLP structure consists of three units of hidden neurons with the sigmoid function. This study [54] indicates that estimated results from MLP are close to the sensor outputs and produced more accurate data for any initial conditions compared with placket model as in Error! Reference 
source not found. However, a large number of iteration stores in MLP causes a heavy burden and heating problem for microcontroller if it has limited memory of processor [47].

Nonlinear autoregressive neural networks (NARX) MLP trained with Levenberg-Marquardt algorithm has been implemented by Ardeev [51] to identify the attitude dynamics of a quadrotor. The Arducopter was placed on a test stand with a spherical joint to eliminate translational motion in all the three axes during the experiment. The NARX MLP results were compared with distributed time-delay neural networks (DTDNN), adaptive neural fuzzy inference system (ANFIS), polynomial regression, and Continues Time Transfer Functions (CTTF). NARX MLP architecture consists of three hidden neurons with four input and output delays that have been determined using a trial and error method.

Sigmoid and linear have been selected as the activation function in the hidden and output layer, respectively. The results showed that NARX MLP performance is better than CTTF and the training time required for NARX MLP is only 69 second. Even though the performance of the polynomial regression method is better, the NARX MLP can identify dynamics model system accurately as in Error! Reference source not found.. It does not require any input selection and is easy to implement into the quadrotor system [51].

Sonntag [50] has implemented a black box MLP structure for identifying the dynamics model of Link Quad quadcopter. Proposed MLP architecture consists of 10 hidden neuron networks and is comparable to a linear grey box approach. Grey box method was implemented by deriving the equations from the first principle modeling and measures directly the parameter constants. This study indicates that the MLP NN can give good predictions for the roll and pitch with noise and the trends in the inputs. It proved that the black box model managed to learn the dynamics of the quadcopter, noise, and trends as in Error! Reference source not found.

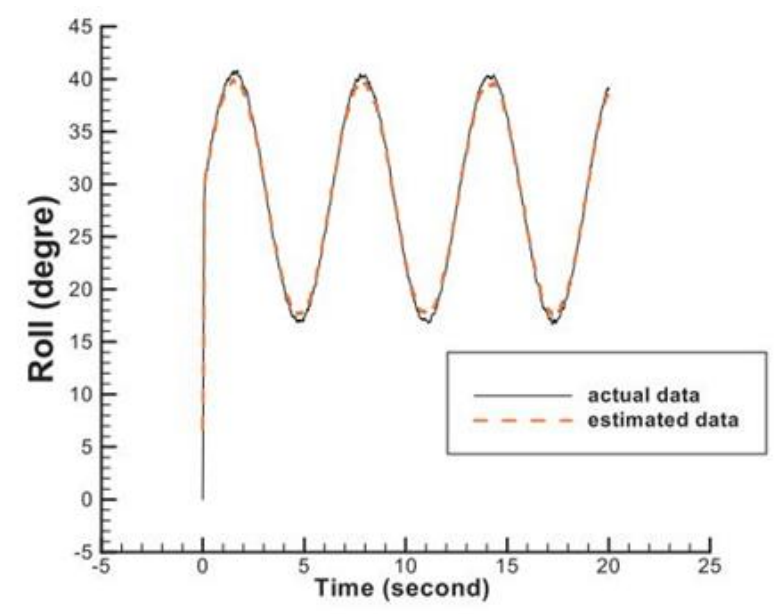

Fig. 9 - The estimated roll angle from the neural network model [54]

The MLP structure with 100 units of hidden neuron had been used by Bansal et al. [52] to find Crazyflie 2.0 quadrotor dynamics model and to prove the ability of NN to learn a dynamic model as shown in Error! Reference source not found. MLP has been trained with a resilient backpropagation learning algorithm. The model obtained from MLP system identification had been used to design a controller for stabilizing the quadcopter in rotational and translational motions. Bansal et al. [52] agreed that a simple two-layer FNN structure feed with the current state and input data identified successfully and learned quadrotor dynamics with high accuracy. Bansal et al. [52] suggested that with proper selection of the NN architecture and inputs, the NN model can identify dynamics model accurately and presents a good alternative for system identification of quadrotor. 

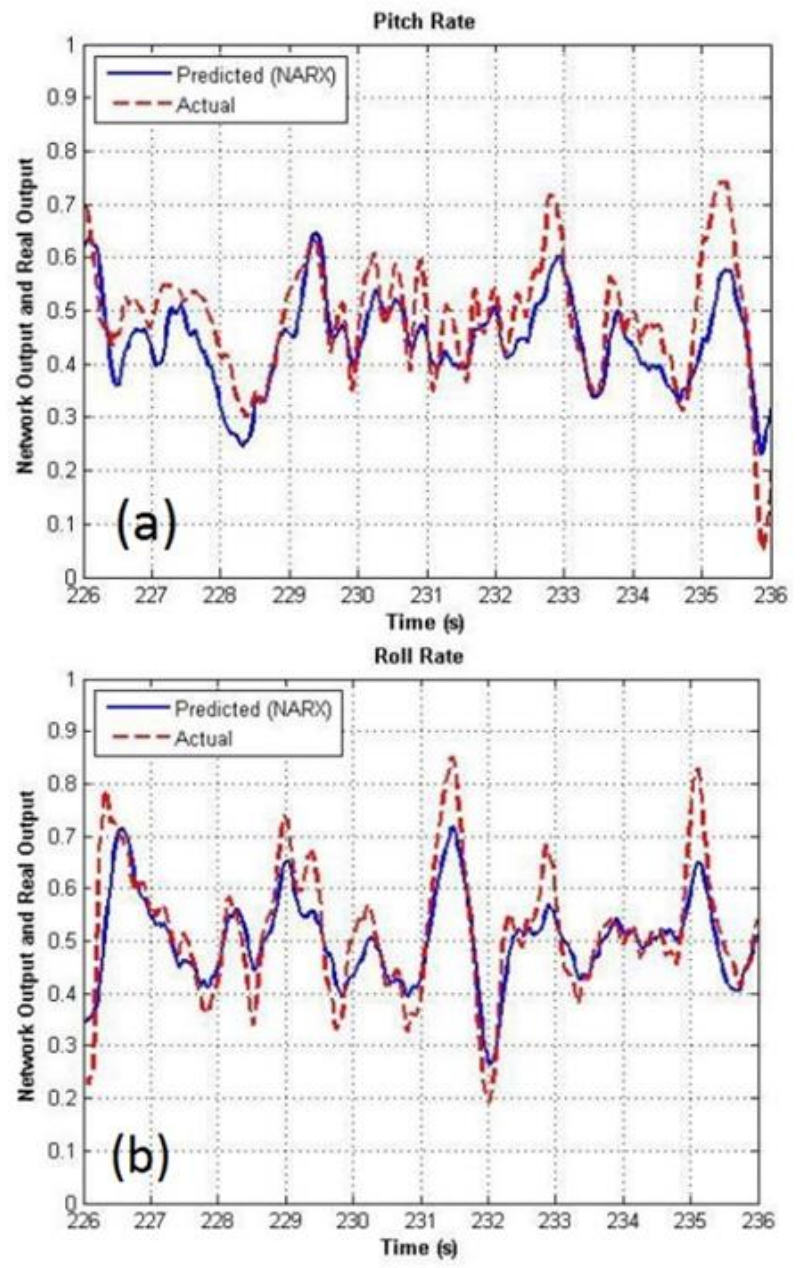

Fig. 10 - Prediction pitch and roll rate for NARX MLP [51] (a) Pitch rate (b) Roll rate

Dakrory and Tawfik [55] compared the MLP NN based model with backpropagation learning technique with the regression least square method. The NN structure used for quadcopter identification consists of two hidden layers. The first and second hidden layers consist of 190 unit and 90 unit neurons, respectively. The MISO NN model consists of 16 inputs and a single output which is roll angle. The researchers selected a single time delay in NARX model to reduce computational time. All the estimation presented in this study is based on running MLP on PC with Intel I7 Processor and Raspberry Pi. The results showed that NN could predict the dynamic systems with good accuracy, much better than the regression method but with longer time for identification, that is, it takes 15.59 minutes with error $0.1916 \%$ to run in $\mathrm{PC}$ while 715.54 minutes is required to run on Raspberrypi.

Recent research by Muliadi and Kusumoputro [56] implement the MLP network structure to model 6 DOF quadrotor dynamics. Authors [56] selected a total of 20 and 4 unit neurons in the hidden and output layer, respectively. Proposed MLP structure will be trained with the backpropagation method to obtain the quadcopter model using two inputs and two outputs as training data. The four minutes of flight test gathering have been conducted to obtain the quadrotor ANN model. The flight has been conducted for rolling and pitching motion while in hovering phase. The recorded flight data have been used to train the MLP to obtain the dynamics model. Dynamics model obtained from the proposed MLP will assess the Artificial Neural Network's Direct Inverse Control (DIC-ANN) and PID controllers. The authors implement the simultaneous twin ANN in simulation for comparison both controller's performance purpose. The simultaneous simulation used ramp and steady state response to excite the dynamics model of quadrotor. The results shown that the MLP NN model are effective to be implement on quadrotor altitude dynamics. 

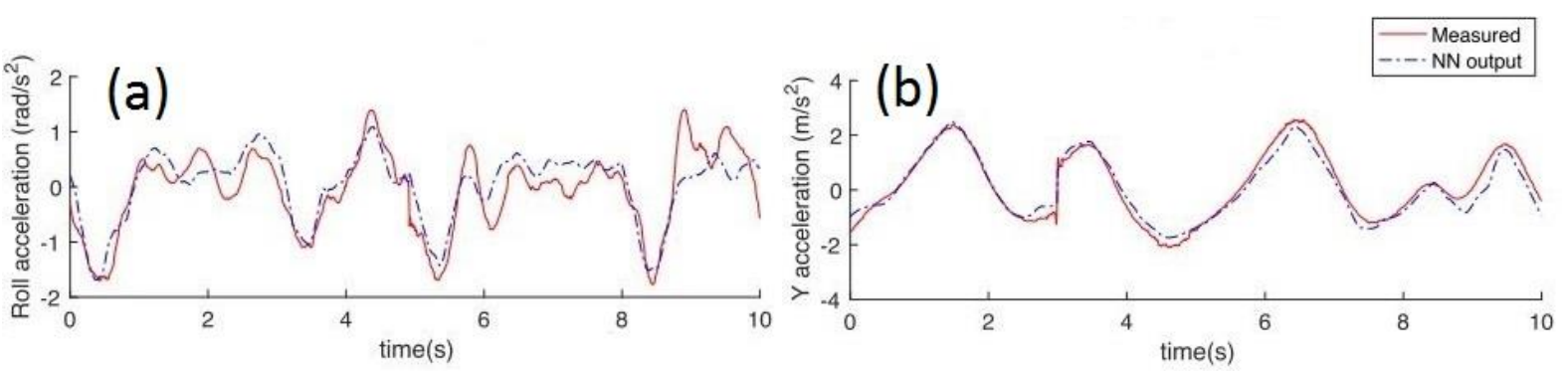

Fig. 11 - Estimation of quadcopter output [52] (a) Roll acceleration (b) y-axis acceleration

\section{Observation 1}

Various strategies can be used to select the numbers of hidden neurons in the MLP network [50], [52], [56]. Majority of past research work used a fixed number of neurons in the MLP network obtained through trial and error procedures while avoiding an excessive number of neurons used to avoid increasing the training time. We believe that very few researches in quadcopter modeling consider the effect of the regression vector and the total number of neurons towards the generalization capability of the proposed NN model.

The overfitting occurred due to the excessive numbers of neurons network matches the data closely and strictly follow the data. Hence, $\mathrm{NN}$ will respond very poorly for new patterns never used in training and lose its generalization ability [23], [38], [57]. The good NN model should have a small number of neurons as possible to obtain a reasonable training error and good generalization abilities [14][57]. Several methods can be used to identify the near-optimal or optimal network structure of the NN model that can be used to achieve good generalization of the prediction model such as K-cross validation [24][45][55] and Lipschitz coefficient [24][58].

An optimized neural network structure criterion strongly related to the size of the regression vector and a total number of hidden neurons in the network. Shamsudin \& Chen [24] implemented a Lipschitz criterion and k-fold cross-validation methods on helicopter UAV to achieve optimized NN structure with appropriated regression vector and hidden neurons size. Another proposed method to achieve near-optimal or optimal network structure is by using cascaded modelling to decrease the amount of weight to be estimated and increase speed [59] [56]. In summary, most of the studies mentioned above agreed that system identification based on the MLP structure has successfully identified the dynamics and system parameters of a quadcopter with acceptable accuracy.

\subsection{Radial Basis Function (RBF)}

RBF network is another type of feedforward NN consisting of a nonlinear hidden layer and a linear output layer that uses basis functions as activation functions in the hidden layer. Basis functions contain parameter vector, center, and calculation of the Euclidean distance between the center and the network input vector. The output of the RBF is a linear combination of the outputs from its hidden units function. RBF architecture shown in Error! Reference source not found. is consists of an input layer, one hidden layer, and an output layer.

Numerous basis functions can be used as the activation function in the hidden neurons, such as thin-plate-spline function, multiquadric function, inverse multiquadric function, and Gaussian function. Theoretical results from Chen and Billings [60] have proved that the selection of the nonlinear activation function is not crucial for RBF. The Gaussian function is the typical choice used due to its suitability for generalizing a global mapping and refining local features without much alteration in the already learned mapping [61]. The output calculation from the RBF structure represents a single hidden layer for RBF network as exemplified in Error! Reference source not found.:

$$
\hat{y}(t \mid \theta)=b_{i}+\sum_{h=1}^{H} w 1_{i h}\left(\exp \left(-\frac{1}{\sigma_{h}^{2}}\|x-\mu\|^{2}\right)\right)
$$

with $\mathrm{h}=1,2,3, \ldots H$ and $\mathrm{i}=1,2,3, \ldots n$ where $w 1_{i h}$ is the weights between the hidden layer and the output layer. Symbol $\mathrm{H}$ denotes the number of neurons in the hidden layer, while $b_{i}$ is the bias elements for the output layer. $\mu$ is the center of the hidden layer, and $\sigma$ is the width of the Gaussian function. The number of inputs and outputs of the neural network is presented by $m$ and $n$, respectively. 


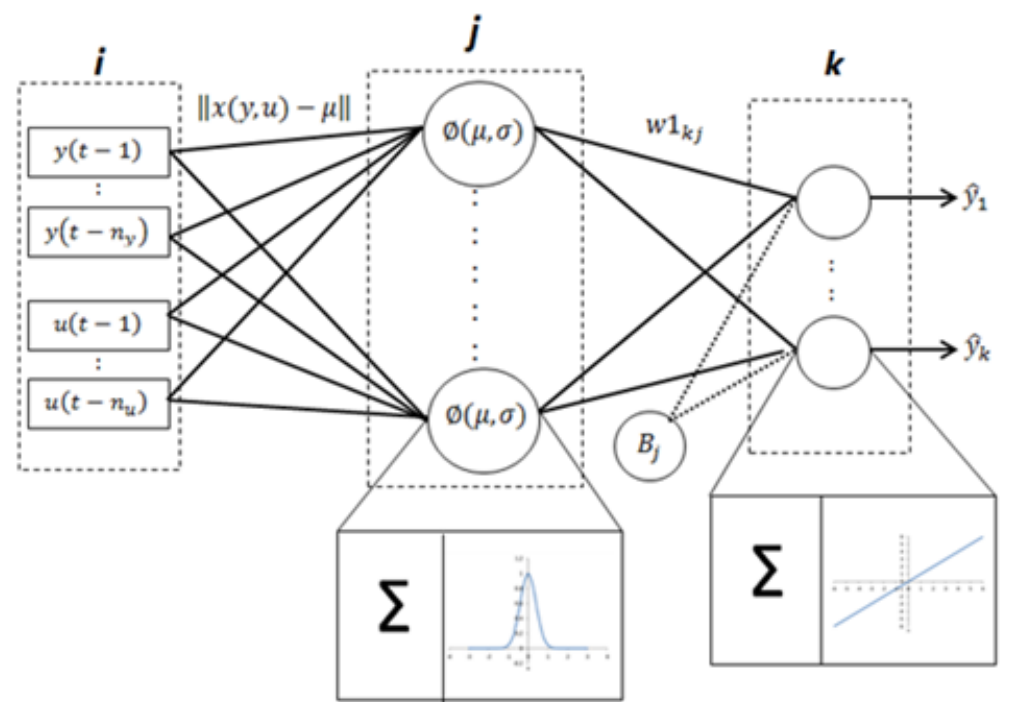

Fig. 12- Basic RBF NN structure

The RBF offers significant prediction qualities improvement compared to traditional MLP [60], [62]. RBF has the capabilities of the local specialization and the global generalization [24]. RBF also has the capability of acting as a universal approximator which is promising for identifying and controlling the dynamic systems [24].

Recent research by Zhong et al. [63] uses RBF NN structure for system identification of quadrotor. The RBF structure proposed in this study consists of four input data, six output data, and seven hidden neurons. The RBF is trained with gradient descent method for updating weights of RBF combined with adaptive learning rate and Welsch function criterion. Adaptive learning rate and Welsch function introduced in RBF training could reduce the effects of the occurrence of large errors and has better performance than other error functions [63].

The result from the proposed trained algorithm is compared with RBF trained with gradient descent learning based on the least mean square (LMS) criterion in a simulation experiment. Both methods showed good predictions of the quadcopter dynamics model. However, RBF with LMS training was impudence by noise disturbance. Both simulation and experiment results from proposed RBF structure can identify with high accuracy and robustness the noise disturbances for quadcopter system as shown in Fig. 13.

In $\mathrm{Wu}$ et al.'s study [49], RBF-ARX model trained with the least square method was proposed to represent the nonlinear dynamics of the quadcopter, and the results were compared with the physical model-based method. The quadrotor used in this study [49] has different configurations with the conventional quadcopter to reduce control complexity and avoid system damage. It has three propellers positioning horizontal to provide pitch and roll motion, and one propeller is mounted in vertical to control yaw motion. Although different configuration, identification result shows that RBF-ARX model is more accurate than the physical model and close to ARX model accuracy with less computation effort. The results revealed that the proposed models could model quadcopter accurately in all 16 working regions.
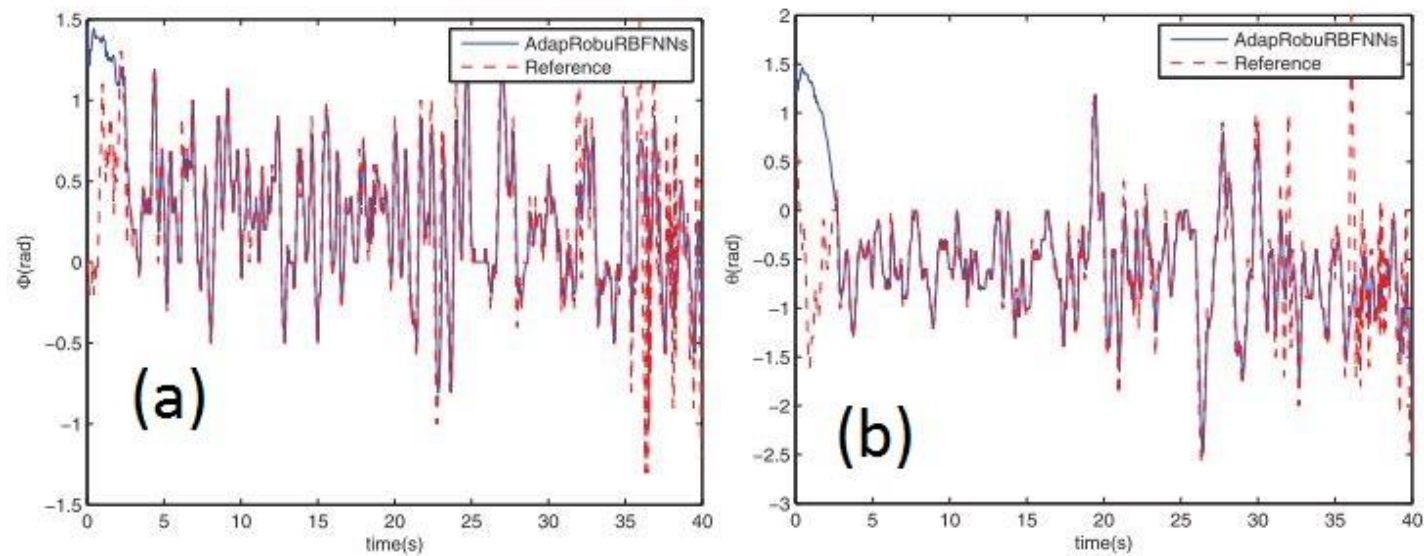

Fig. 13 - Prediction of quadcopter output [63] (a) Pitch rate (b) Roll rate 


\section{Observation 2}

Several findings in the different area of flight dynamic modelling suggest that RBF networks produce improved prediction accuracy with faster training time compared to MLP network trained with backpropagation algorithm [64][65][66]. We believe that more comparative studies need to conducted in the future to validate the efficiency of the RBF network in the case of quadcopter modelling. The modelling studies need to include basic evaluation to select the proper number of neurons, center and centroid to avoid over-fitting error in modelling. The proposed method to achieve an optimized RBF structure can be done by implementing the fully tuned RBF network method to automatically selected the RBF parameters [67][68]. To implement the fully tuned RBF neural network, more sophisticated and highperformance microcontroller was required. The high-performance microcontroller is needed so that RBF can operate within the sampling time required. Typically, batch training implementation in NN learning method should provide good prediction accuracy compared to online training. However, if training data is large, batch training method requires more training time. Several researchers have proposed mini-batch training approach to train the NN model with large data to minimize training time. The large training data would be split into a smaller number of data samples for the mini-batch offline training. However, these techniques can only be used on relatively small networks because of the finite amount of computing power available in the real-time processor and are limited to uncoupled dynamics models.

\section{Conclusion}

In conclusion, neural networks provided an excellent alternative for system identification of quadrotor, especially when it is difficult to derive a physical-based model of the system. NN based system identification is capable of obtaining the dynamics of a quadcopter with high accuracy while reducing the development time, costs and resources for modelling. If users make any modifications to the quadcopter, the NN will be able to learn and estimate the action of the quadcopter. Based on the review on NN based system identification, most researchers focus on the application of MLP structure for quadcopter dynamic modeling. Moreover, there is an opportunity for future research in the implementation of RBF network or any other advanced structure of NN such as the hybrid multilayer perceptron (HMLP) and direct-link radial basis function (DRBF) for system identification of quadcopter dynamics.

\section{Acknowledgement}

This research is support by Universiti Tun Hussien Onn Malaysia under the Geran Penyelidikan Pascasiswazah (GPPS) Vot H364

\section{References}

[1] G. Cai, J. Dias, and L. Seneviratne, A Survey of Small-Scale Unmanned Aerial Vehicles : Recent Advances and Future Development Trends, Unmanned Syst., vol. 2, no. 2, pp. 1-25, 2014

[2] W. Wei, Development of an Effective System Identification and Control Capability for Quadcopter UAVs, University of Cincinnati, 2015

[3] A. Puri, A Survey of Unmanned Aerial Vehicles (UAV) for Traffic Surveillance, Tech. Pap., pp. 1-29, 2005

[4] E. Altuğ, J. P. Ostrowski, and C. J. Taylor, Control of a Quadrotor Helicopter Using Dual Camera Visual Feedback, Int. J. Rob. Res., vol. 24, no. 5, pp. 329-341, 2005

[5] N. V. Hoffer, C. Coopmans, A. M. Jensen, and Y. Chen, A survey and categorization of small low-cost unmanned aerial vehicle system identification, J. Intell. Robot. Syst., vol. 74, no. 1-2, pp. 129-145, 2014

[6] J. Awang Besar, Quadrotor Stability using PID, Universiti Tun Hussein Onn Malaysia, 2013

[7] M. Q. Vechian, Wireless Control Quadcopter With Stereo Camera and Self-Balancing System, Universiti Tun Hussein Onn Malaysia, 2012

[8] M. F. Ramli, S. S. Shamsudin, and A. Legowo, Safe avoidance path detection using multi sensor integration for small unmanned aerial vehicle, 5th IEEE Int. Work. Metrol. AeroSpace, Metroaerosp. 2018 - Proc., pp. 101-106, 2018

[9] M. F. Ramli, S. S. Shamsudin, and A. Legowo, Obstacle detection technique using multi sensor integration for small unmanned aerial vehicle, Indones. J. Electr. Eng. Comput. Sci., vol. 8, no. 2, pp. 441-449, 2017

[10] M. F. Ramli, A. Legowo, and S. S. Shamsudin, Object Detection Technique for Small Unmanned Aerial Vehicle, IOP Conf. Ser. Mater. Sci. Eng., vol. 260, no. 1, 2017

[11] M. Kemper and S. Fatikow, Impact of center of gravity in quadrotor helicopter controller design, in IFAC Proceedings Volumes, 2006, vol. 39, no. 16, pp. 157-162

[12] M. Bangura, M. Melega, R. Naldi, and R. Mahony, Aerodynamics of Rotor Blades for Quadrotors, arXiv, 2016.

[13] G. Cai, H. Al Mehairi, H. Al-Hosani, J. Dias, and L. Seneviratne, Frequency-domain flight dynamics model identification of MAVs-miniature quad-rotor aerial vehicles, in International Conference on Intelligent Robots and Systems (IROS 2014), 2014, no. Iros, pp. 3376-3381 
[14] S. S. Shamsudin, The Development of Neural Network Based System Identification and Adaptive Flight Control for an Autonomous Helicopter System, 2013

[15] G. Cai, B. M. Chen, and T. H. Lee, Unmanned Rotorcraft Systems. New York: Springer-Verlag London Limited 2011, 2006

[16] G. Cai, T. Taha, J. Dias, and L. Seneviratne, A framework of frequency-domain flight dynamics modeling for multi-rotor aerial vehicles, Proc. Inst. Mech. Eng. Part G J. Aerosp. Eng., vol. 0, no. 0, pp. 1-17, 2016

[17] S. L. Waslander, G. M. Hoffmann, C. J. Tomlin, S. J. Jang, and C. J. Tomlin, Multi-Agent Quadrotor Testbed Control Design : Integral Sliding Mode vs Reinforcement Learning *, Int. Conf. Intell. Robot. Syst., pp. 468-473, 2005

[18] N. Abas, A. Legowo, and R. Akmeliawati, Parameter Identification in quadrotor, in International Conference on Mechatronics (ICOM), 2011, pp. 17-19

[19] I. E. Putro, A. Budiyono, K. J. Yoon, and D. H. Kim, Modeling of unmanned small scale rotorcraft based on neural network identification, in International Conference on Robotics and Biomimetics, 2008, pp. 1938-1943

[20] M. B. . . Tischler and R. K. Remple, Aircraft and Rotorcraft System Identification: Engineering Methods with Flight Test Examples. Reston, Virginia: American Institute of Aeronautics and Astronautics, 2006

[21] V. Manuel, A. Res, and E. R. Araiza, System Identification of a Quad-rotor in X Configuration from Experimental Data, Research Compting Sci., vol. 118, pp. 77-86, 2016

[22] J. M. Zurada, Applications of Neural Networks for Aerospace-Related Technologies, in Aerospace Applications Conference, 1996, pp. 279-286

[23] F. S. Panchal and M. Panchal, Review on Methods of Selecting Number of Hidden Nodes in Artificial Neural Network, Int. J. Comput. Sci. Mob. Comput., vol. 3, no. 11, pp. 455-464, 2014

[24] S. S. Shamsudin and X. Chen, Identification of an unmanned helicopter system using optimized neural network structure, Int. J. Model. Identif. Control, vol. 17, no. 3, pp. 223-241, 2012

[25] S. Gupte, P. I. T. Mohandas, and J. M. Conrad, A survey of quadrotor unmanned aerial vehicles, in Conference Proceedings - IEEE SOUTHEASTCON, 2012, pp. 1-6

[26] P. McKerrow, Modelling the Draganflyer four-rotor helicopter, in IEEE International Conference on Robotics and Automation, 2004, vol. 4, no. May, pp. 3596-3601

[27] M. Bangura and R. Mahony, Nonlinear Dynamic Modeling for High Performance Control of a Quadrotor, Australas. Conf. Robot. Autom. (ACRA 2012), pp. 1-10, 2012

[28] T. Bresciani, Modelling, Identification and Control of a Quadrotor Helicopter, Lund University, 2008

[29] P. Pounds, R. Mahony, J. Gresham, P. Corke, and J. Roberts, Towards Dynamically-Favourable Quad-Rotor Aerial Robots, Australas. Conf. Robot. Autom., p. 10, 2004

[30] X. Zhang, X. Li, K. Wang, and Y. Lu, A survey of modelling and identification of quadrotor robot, Abstr. Appl. Anal., vol. 2014, pp. 1-16, 2014

[31] V. Puttige and S. Anavatti, Real-time system identification of unmanned aerial vehicles: A multi-network approach, J. Comput., vol. 3, no. 7, pp. 31-38, 2008

[32] I. M. Salameh, E. M. Ammar, and T. A. Tutunji, Identification of Quadcopter Hovering Using Experimental Data, in Jordan Conference on Applied Electrical Engineering and Computing Technologies, 2015, pp. 3-8

[33] G. Gremillion and J. Humbert, System Identification of a Quadrotor Micro Air Vehicle, in AIAA Atmospheric Flight Mechanics Conference, 2010

[34] D. S. Miller, Open-loop system identification of a micro quadrotor helicopter from closed-loop data, University of Maryland, 2011

[35] M. G. Yoo, S. K. Hong, and C. Author, Dynamic System Identification and Validation of a Quadrotor UAV, Int. J. Appl. Eng. Res., vol. 11, no. 2, pp. 1089-1093, 2016

[36] G. Cai, B. M. Chen, and T. H. Lee, An overview on development of miniature unmanned rotorcraft systems, Front. Electr. Electron. Eng. China, vol. 5, no. 1, pp. 1-14, 2010

[37] A. S. Imam and R. Bicker, Quadrotor Comprehensive Identification from Frequency Responses, Int. J. Sci. Eng. Res., vol. 5, no. 2, pp. 795-804, 2014

[38] S. Samarasinghe, Neural networks for applied sciences and engineering: from fundamentals to complex pattern recognition. New York: Auerbach Publications, 2007

[39] J. Cordeiro, Using Neural Networks for Identification and Control of Systems, MAICS, pp. 83-89, 2015

[40] R. Kumar, R. Ganguli, and S. N. Omkar, Rotorcraft parameter estimation using radial basis function neural network, Appl. Math. Comput., vol. 216, no. 2, pp. 584-597, 2010

[41] K. Kirkpatrick, J. M. Jr, and J. Valasek, Aircraft System Identification Using Artificial Neural Networks, in AIAA Aerospace Sciences Meeting including the New Horizons Forum and Aerospace Exposition, 2013, no. January, pp. $1-12$

[42] N. K. Peyada and A. K. Ghosh, Aircraft Parameter Estimation using Neural Network based algorithm, in AIAA Atmospheric Flight Mechanics Conference, 2009, no. August, pp. 1-13

[43] B. P. Rimal, I. E. Putro, A. Budiyono, D. Min, and E. Choi, System Identification of NN-based Model Reference Control of RUAV during Hover, in Artificial Neural Networks - Industrial and Control Engineering Applications, 
INTECH, 2016, pp. 395-420

[44] M. Samal, Neural network based identification and control of an unmanned helicopter, University of New South Wales, 2009

[45] S. S. Shamsudin and X. Chen, Recursive Gauss-Newton based training algorithm for neural network modelling of an unmanned rotorcraft dynamics, Int. J. Intell. Syst. Technol. Appl., vol. 13, pp. 56-80, 2014

[46] M. Lawryńczuk, Computationally Efficient Model Predictive Control Algorithms. Warsaw: Springer International Publishing, Switzerland, 2014

[47] T. N. Dief and S. Yoshida, Review: Modeling and Classical Controller of Quadrotor, IRACST - Int. J. Comput. Sci. Inf. Technol. Secur., vol. 5, no. August, pp. 314-319, 2015

[48] J. O. Pedro and A. J. Crouse, Direct Adaptive Neural Control of a Quadrotor Unmanned Aerial Vehicle, in Control Conference (ASCC), 2015, pp. 1-6.

[49] J. Wu, H. Peng, Q. Chen, and X. Peng, Modeling and control approach to a distinctive quadrotor helicopter, ISA Trans., vol. 53, no. 1, pp. 173-185, 2014

[50] D. Sonntag, A Study of Quadrotor Modelling, Linköpings University, 2011

[51] A. Avdeev, Artificial Intelligence based Identification of the Attitude Dynamics for a Quadrotor UAV, University of Sharjah, 2014

[52] S. Bansal, F. J. Jiang, C. J. Tomlin, A. K. Akametalu, and F. Laine, Learning Quadrotor Dynamics Using Neural Network for Flight Control, Conf. Decis. Control 2016, vol. 1610.05863, 2016

[53] A. Vargas, M. Ireland, and D. Anderson, System Identification of Multi-Rotor UAV's Using Echo State Networks, in AUVSI's Unmanned Systems, 2015, pp. 1-11

[54] T. N. Dief and S. Yoshida, System Identification for Quad-rotor Parameters Using Neural Network, Evergr. Jt. J. Nov. Carbon Resour. Sci. Green Asia Strateg., vol. 03, no. 01, pp. 6-11, 2016

[55] A. . ELDakrory and M. Tawfik, Identifying the Attitude of Dynamic systems using Neural Network., in International Workshop on Recent Advances in Robotics and Sensor Technology for Humanitarian Demining and Counter-IEDs (RST), 2016, pp. 1-4

[56] J. Muliadi and B. Kusumoputro, Neural Network Control System of UAV Altitude Dynamics and Its Comparison with the PID Control System, J. Adv. Transp., vol. 2018, no. D, pp. 1-18, 2017

[57] D. Hunter, H. Yu, S. Member, M. S. Pukish, J. Kolbusz, and B. M. Wilamowski, Selection of Proper Neural Network Sizes and Architectures - A Comparative Study, IEEE Trans. Ind. Informatics, vol. 8, no. 2, pp. 228240,2012

[58] N. Mohajerin and S. L. Waslander, Modular deep recurrent neural network: Application to quadrotors, in IEEE International Conference on Systems, Man and Cybernetics, 2014, pp. 1374-1379

[59] J. Zhong, T. Lei, and G. Yao, Robust vehicle detection in aerial images based on cascaded convolutional neural networks, Sensors (Switzerland), vol. 17, no. 12, 2017

[60] S. Chen and S. a. Billings, Neural networks for nonlinear dynamic system modelling and identification, Int. J. Control, vol. 56, no. 2, pp. 319-346, 1992

[61] N. Sundararajan, P. Saratchandran, and Van Li, Fully Tuned Radial Basis Function Neural Networks For Flight Control, 1st ed. Singapore: Kluwer Academic Publishers, 2002

[62] T. Xie, H. Yu, and B. Wilamowski, Comparison between traditional neural networks and radial basis function networks, in International Symposium on Industrial Electronics, 2011, no. July 2011, pp. 1194-1199

[63] H. Zhong, S. Li, Y. Wang, and H. Liu, Adaptive Robust RBFNNs-based Model Estimator for a Small Quadrotor Aircraft Robot, in International Conference on Mechatronic and Embedded Systems and Applications (MESA), 2016

[64] M. A. Ghorbani, H. A. Zadeh, M. Isazadeh, and O. Terzi, A comparative study of artificial neural network ( MLP, RBF ) and support vector machine models for river flow prediction, Environ. Earth Sci., vol. 75, no. 6, pp. 1-14, 2016

[65] A. W. Jayawardena, D. A. . Fernando, and M. . Zhou, Comparison of multilayer perceptron and radial basis function networks as tools for flood forecasting, in Destructive Water: Water-Caused Natural Disasters, their Abatement and Control, 1997, no. 239, pp. 173-181

[66] L. Jasim Saud and Z. Kudair Abass, A Comparison between Multi-Layer Perceptron and Radial Basis Function Networks in Detecting Humans Based on Object Shape, Ibn AL-Haitham J. Pure Appl. Sci., vol. 31, no. 2, p. 210,2018

[67] M. F. Pairan and S. S. Shamsudin, System identification of an unmanned quadcopter system using MRAN neural, in IOP Conference Series: Materials Science and Engineering, 2017, vol. 270, pp. 1-10

[68] P. Chandrakumar, P. Saratchandran, and N. Sundararajan, Minimal radial basis function neural networks for nonlinear channel equalization, IEE Vis. Image Signal Process., vol. 147, no. 5, pp. 428-435, 2000. 